\title{
Successful treatment of central retinal artery occlusion using hyperbaric oxygen therapy
}

\author{
Soo Han Kim², Yong Sung Cha ${ }^{2}$, Yoonsuk Lee ${ }^{2}$, Hyun Kim², le Na Yoon ${ }^{1}$ \\ 'Department of Ophthalmology, Yonsei University Wonju College of Medicine, Wonju, Korea \\ ${ }^{2}$ Department of Emergency Medicine, Yonsei University Wonju College of Medicine, Wonju, Korea
}

Central retinal artery occlusion (CRAO) is considered an ophthalmologic emergency. The prognosis of this disease is very poor. Currently, there is no generally effective therapy available to treat CRAO. Hyperbaric oxygen therapy (HBOT) can increase the volume of oxygen delivered to the ischemic retinal tissue until spontaneous or assisted reperfusion occurs. We report the case of a patient who experienced sudden visual loss due to CRAO that was treated with HBOT. The patient was an 81-year-old woman who presented with CRAO in her right eye (OD). She exhibited "hand motion" visual acuity before treatment. She underwent three sessions of HBOT at a pressure of 2.8 atmospheres absolute, performed over 3 days. After 4 days in hospital, her visual acuity improved to 0.4 (OD) for far vision and 0.5 (OD) for near vision. Her vision was stable without the supply of oxygen; therefore, she was discharged.

Keywords Retinal artery occlusion; Hyperbaric oxygenation; Ophthalmic artery

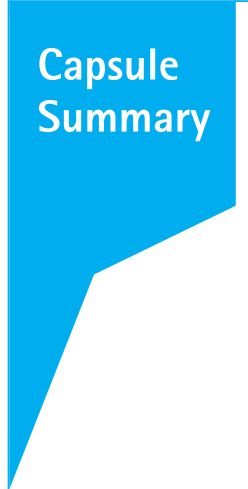

eISSN: 2383-4625

Received: September 82017

Revised: October 272017

Accepted: October 302017

Correspondence to: Yong Sung Cha Department of Emergency Medicine, Yonsei University Wonju College of Medicine, 20 Ilsan-ro, Wonju 26426, Korea

E-mail: emyscha@yonsei.ac.kr ORCID

http://orcid.org/0000-0001-9897-4273

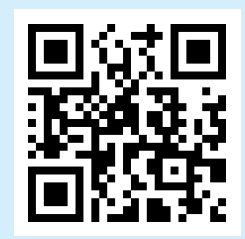

What is already known

Central retinal artery occlusion (CRAO) treatments focus on moving the embolus downstream by lowering intraocular pressure and producing vasodilatation. However, there are currently no generally effective therapies available for CRAO patients.

What is new in the current study

In CRAO, hyperbaric oxygen therapy enhances oxygen delivery to the ischemic retinal tissue until either spontaneous or assisted reperfusion occurs.
How to cite this article:

Kim SH, Cha YS, Lee Y, Kim H, Yoon IN. Successful treatment of central retinal artery occlusion using hyperbaric oxygen therapy. Clin Exp Emerg Med 2018;5(4):278281.

This is an Open Access article distributed under the terms of the Creative Commons Attribution Non-Commercial License (http:// creativecommons.org/licenses/by-nc/4.0/). 


\section{INTRODUCTION}

Central retinal artery occlusion (CRAO) is a devastating and common ophthalmologic condition. ' CRAO presents as a sudden, unilateral, and painless loss of vision. ${ }^{2,3}$ Even when treated promptly, an acute obstruction of the central retinal artery usually results in severe and permanent loss of vision. ${ }^{4,5}$

Traditional CRAO treatments (ocular massage, anterior chamber paracentesis, intraocular pressure-lowering medications, vasodilators, and oral diuretics) focus on moving the embolus downstream by lowering intraocular pressure and producing vasodilatation. ${ }^{5,6}$ However, there are currently no effective therapies available for CRAO. ${ }^{5-7}$

Another treatment for CRAO is hyperbaric oxygen therapy (HBOT). This involves the inhalation of 100\% oxygen at pressures exceeding 1 atmosphere absolute (ATA). During HBOT, the volume of oxygen dissolved in the plasma increases by up to 20 to 30 times. ${ }^{8}$ We report the first case of successful treatment of CRAO through early HBOT in Korea.

\section{CASE REPORT}

An 81-year-old woman presented at the emergency department (ED) with a sudden, painless loss of vision in the right eye (OD). Her visual change had started 10 hours prior to ED admission. The patient's medical history revealed heart failure, atrial fibrillation, and a renal infarct. She had undergone cataract surgery on both eyes.

When the patient visited the ED, her non-corrected visual acuity was noted to be hand motion only in the $\mathrm{OD}$ and 0.4 on a decimal scale in the left eye (OS). The intraocular pressure was 14 $\mathrm{mmHg}$ in the OD and $13 \mathrm{mmHg}$ in the OS. She exhibited a grade 3 relative afferent pupillary defect in her OD. The anterior segment of her eyes was evaluated using biomicroscopy, with no abnormal findings. A dilated fundus examination revealed a slightly pale retina with a cherry-red spot in the macula. No definite evidence of a plaque or embolus was noted. A domain optical coherence tomography scan showed that there was a mild increase in the reflectivity of the inner retinal layer (Fig. 1A). The patient was instructed on how to perform a digital massage on her OD. Topical brimonidine and dorzolamide/timolol were also prescribed to maximize the perfusion pressure.

Following the diagnosis of CRAO in the ED, we initiated oxygen therapy for 30 minutes via a facial mask with a reservoir bag (15 L/min), with no significant improvement in the patient's vision. Therefore, we decided to treat the patient with HBOT. Her vision failed to improve significantly at pressures of 2 and 2.4 ATA, and then, compressed to 2.8 ATA. The patient indicated that her vision
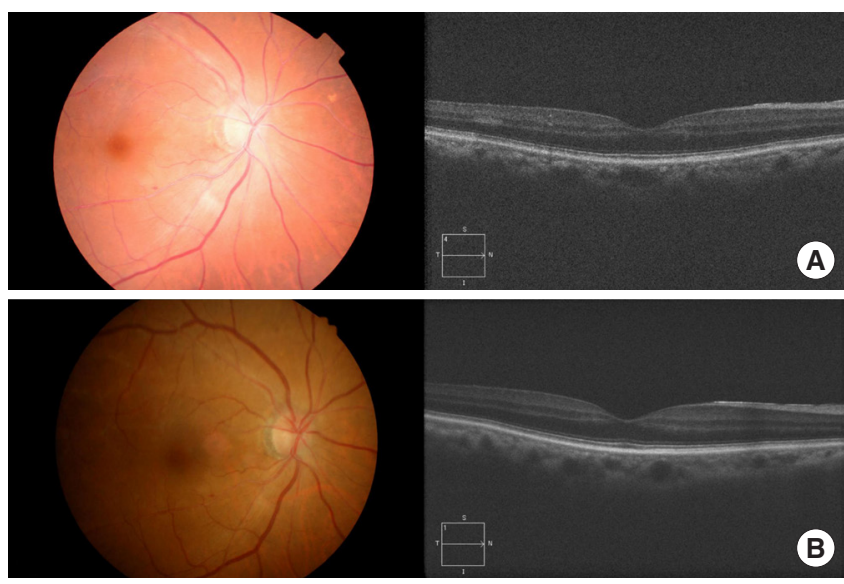

Fig. 1. Fundus photographs and optical coherence tomography imaging both (A) before and $(B)$ after the patient underwent hyperbaric oxygen therapy.

comprised similar clarity as that prior to the CRAO diagnosis at 2.8 ATA, so we continued the treatment at a pressure of 2.8 ATA for 140 minutes in the multiplace chamber (IBEX Medical Systems, Seoul, Korea) (Fig. 2). After the first session, the patient's non-corrected visual acuity improved to 0.2 in the $\mathrm{OD}$ and 0.5 in the OS. After her admission, she received intermittent oxygen therapy for 15 minutes every hour, alternating with 45 minutes of breathing room air, followed by an ophthalmologist performing anterior chamber paracentesis. After anterior chamber paracentesis, her visual acuity did not improve. The patient was also administered a $6 \mathrm{~L} / \mathrm{min}$ supply of oxygen while sleeping. If her visual acuity decreased, we planned to restart the HBOT. The next day, the patient exhibited prompt light reflexes without relative afferent pupillary defect and presented with a non-corrected vision of 0.4 in the OD and 0.6 in the OS. When examined, it was noted that the cherry-red spot was no longer visible on the fundus, and increased reflectivity and irregular margins of the inner retinal layer were not observed (Fig. 1B). During the HBOT session on the second day, the ophthalmologist was present to monitor visual improvement during the HBOT (Fig. 3). Her near visual acuity in the OD gradually increased during the HBOT from 0.1 to 0.5 . She received one session of HBOT (with the same treatment protocol for each session) per day for 3 days, with intermittent oxygen therapy following the HBOT. No complications occurred during HBOT. On the fourth day, the patient's visual acuity remained 0.4 (OD) for her far vision and 0.5 (OD) for near vision, the same as that without an oxygen supply (Fig. 4). The patient was then discharged. She was followed up one month after discharge at the ophthalmology clinic, and her visual acuity was maintained at 0.8 (OD) without any complications, such as neovascularization or retinal pigment epithelium changes. 


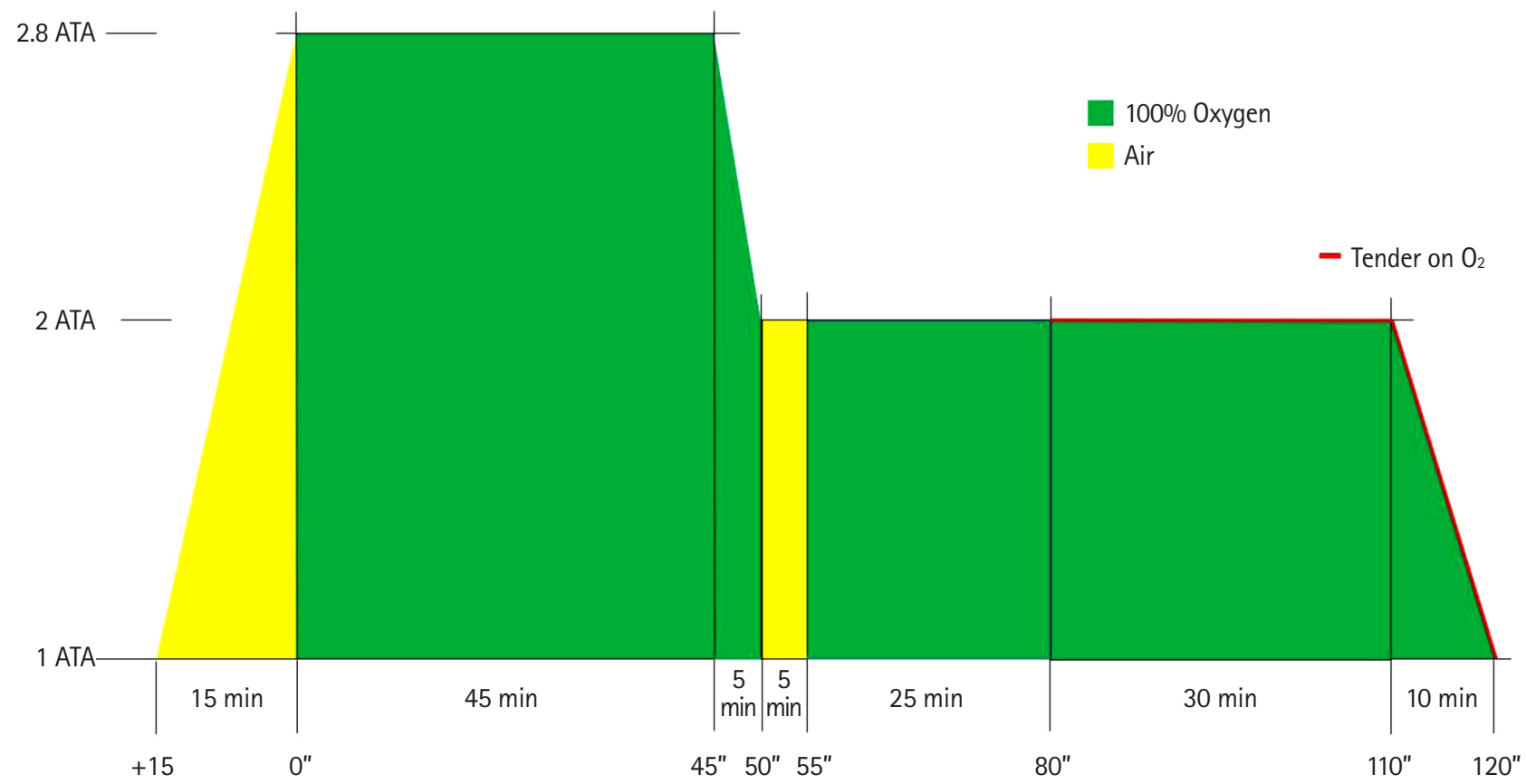

Fig. 2. Overview of initial treatment with hyperbaric oxygen therapy applied to the patient. ATA, atmosphere absolute.

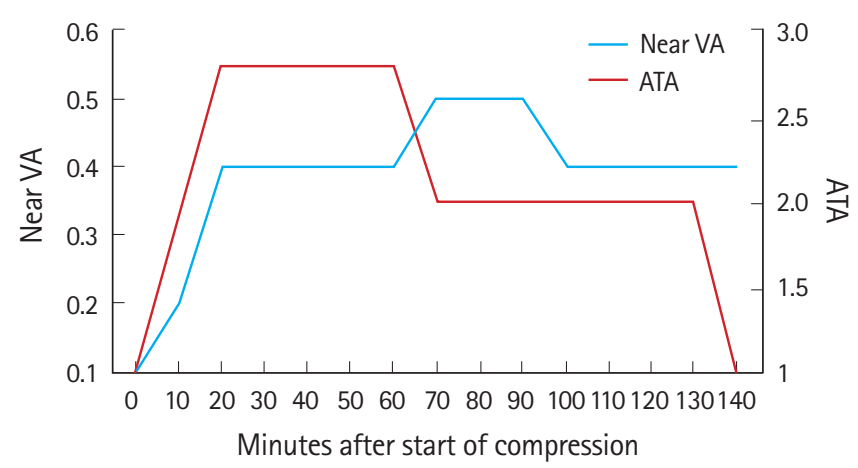

Fig. 3. Changes in the patient's near visual acuity (VA) during hyperbaric oxygen therapy. ATA, atmosphere absolute.

\section{DISCUSSION}

The key reason for the poor prognosis following a CRAO diagnosis is that the retina is very sensitive to ischemia, as retinal tissue demonstrates the highest oxygen consumption rate per unit mass in the human body. ${ }^{9}$ The inner retinal layers, which are normally served by retinal circulation, typically lose viability following an occlusion, causing vision loss among CRAO patients; however, if these layers can obtain enough oxygen via diffusion through the choroidal circulation, the inner retinal layers will remain viable. ${ }^{10}$ Oxygenation of the ischemic inner retinal layers by HBOT depends on the choroid perfusion that occurs under CRAO conditions. ${ }^{11}$ If the level of occlusion is at the ophthalmic artery, the blood sup-

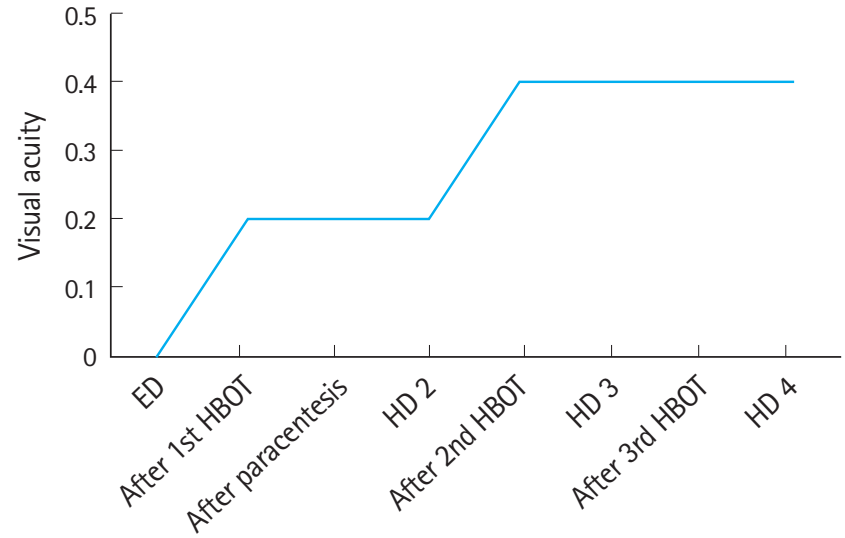

Fig. 4. Overall changes in the patient's visual acuity in her right eye. ED, emergency department; HBOT, hyperbaric oxygen therapy; HD, hospital admission day.

ply to the posterior ciliary vessels will also be blocked, and there will be no collateral circulation to oxygenate the inner retina. ${ }^{12}$ An adequate partial pressure of oxygen should be maintained in order to keep the retina viable until circulation is restored via natural recanalization, which usually occurs within 72 hours. ${ }^{13}$ As no single modality is sufficient to treat CRAO, multiple treatments were administered in this case to maintain the patient's vision. Notably, there was an immediate visual improvement after the first session of HBOT and an improvement in the patient's near vision after the second HBOT session, suggesting that the 
patient's visual improvement was likely due to the HBOT. There are several limitations in this case presentation. First, fluorescein angiography was not performed. Therefore, the exact severity of the circulation defect could not be assessed. Second, multiple treatment modalities were applied to the patient, as no single modality is sufficient to treat CRAO. Although she did not experience any visual improvement during other treatments, there is a possibility that her vision was also aided from other modalities.

The Undersea and Hyperbaric Medical Society has recommended that patients presenting for treatment within 24 hours after symptom onset should be considered for HBOT. ${ }^{13}$ However, as medical insurance currently does not cover HBOT use to treat CRAO in Korea, we recommend that further studies be carried out to evaluate the effects of HBOT among Korean patients with CRAO.

\section{CONFLICT OF INTEREST}

No potential conflict of interest relevant to this article was reported.

\section{REFERENCES}

1. Rumelt $S$, Dorenboim $Y$, Rehany U. Aggressive systematic treatment for central retinal artery occlusion. Am J Ophthalmol 1999;128:733-8.

2. Augsburger JJ, Magargal LE. Visual prognosis following treatment of acute central retinal artery obstruction. $\mathrm{Br} J$ Ophthalmol 1980;64:913-7.

3. Karjalainen K. Occlusion of the central retinal artery and retinal branch arterioles: a clinical, tonographic and fluorescein angiographic study of 175 patients. Acta Ophthalmol Suppl
1971;109:1-95.

4. Hayreh SS, Zimmerman MB. Central retinal artery occlusion: visual outcome. Am J Ophthalmol 2005;140:376-91.

5. Duker JS, Brown GC. Recovery following acute obstruction of the retinal and choroidal circulations: a case history. Retina 1988;8:257-60.

6. Stone $R$, Zink $H$, Klingele $T$, Burde RM. Visual recovery after central retinal artery occlusion: two cases. Ann Ophthalmol 1977:9:445-50.

7. Neubauer AS, Mueller AJ, Schriever S, Gruterich M, Ulbig M, Kampik A. Minimally invasive therapy for clinically complete central retinal artery occlusion: results and meta-analysis of literature. Klin Monbl Augenheilkd 2000;217:30-6.

8. Fosen KM, Thom SR. Hyperbaric oxygen, vasculogenic stem cells, and wound healing. Antioxid Redox Signal 2014;21: 1634-47.

9. Hayreh SS, Zimmerman MB, Kimura A, Sanon A. Central retinal artery occlusion: retinal survival time. Exp Eye Res 2004; 78:723-36.

10. Patz A. Oxygen inhalation in retinal arterial occlusion: a preliminary report. Am J Ophthalmol 1955;40:789-95.

11. Li HK, Dejean BJ, Tang RA. Reversal of visual loss with hyperbaric oxygen treatment in a patient with Susac syndrome. Ophthalmology 1996;103:2091-8.

12. Murphy-Lavoie $H$, Butler $F$, Hagan C. Central retinal artery occlusion treated with oxygen: a literature review and treatment algorithm. Undersea Hyperb Med 2012;39:943-53.

13. Murphy-Lavoie $H$, Butler $F$, Hagan C. Arterial inefficiencies: central renal artery occlusion. In: Lindell KW, editor. Hyperbaric oxygen therapy indication. 13th ed. Durham, NC: Best Publishing Company; 2014. p.11-24. 